\section{The AM-GM Inequality is Equivalent to the Bernoulli Inequality}

\section{Lech Maligranda}

$T$

he AM-GM (= arithmetic-mean/geometric-mean) inequality is sometimes called the Cauchy inequality (1821):

$$
A_{n}=\frac{x_{1}+\cdots+x_{n}}{n} \geq\left(x_{1} \cdot \ldots \cdot x_{n}\right)^{1 / n}=G_{n} \quad\left(\text { all } x_{k}>0\right) .
$$

In the books [1] and [2] there are given 52 and 74 proofs of this inequality, respectively, and mathematicians still find new proofs (!).

Much earlier, Isaac Barrow (1670) and Jacob Bernoulli (1689) proved the inequality, which now has the name Bernoulli inequality:

$$
x^{n} \geq 1+n(x-1) \text { for any } x>0 \text { and } n \in \mathbb{N} .
$$

In the paper [5] we can read about the history, generalizations, and different proofs of the Bernoulli inequality.

In this note I show that these inequalities are in fact equivalent, that is, each follows one from the other. This was observed in [4, Thm 3] (see also [2], p. 213, and [5, Thm $10]$ ), but I present here even simpler arguments.

TheOrem Inequalities (AG) and (B) are equivalent.

Proof. (B) $\Longrightarrow\left(\right.$ AG). Since $\frac{A_{n}}{A_{n-1}}>0$ it follows from the Bernoulli inequality (B) that

$$
\begin{aligned}
\left(\frac{A_{n}}{A_{n-1}}\right)^{n} & \geq 1+n\left(\frac{A_{n}}{A_{n-1}}-1\right) \\
& =\frac{A_{n-1}+n A_{n}-n A_{n-1}}{A_{n-1}} \\
& =\frac{n A_{n}-(n-1) A_{n-1}}{A_{n-1}}=\frac{x_{n}}{A_{n-1}}
\end{aligned}
$$

or

$$
A_{n}^{n} \geq x_{n} \cdot A_{n-1}^{n-1} .
$$

Now, using inequality (C) successively, we obtain

$$
\begin{aligned}
A_{n}^{n} & \geq x_{n} \cdot A_{n-1}^{n-1} \geq x_{n} \cdot x_{n-1} \cdot A_{n-2}^{n-2} \\
& \geq \ldots \geq x_{n} \cdot x_{n-1} \cdot \ldots x_{2} \cdot A_{1}^{1} \\
& =x_{n} \cdot x_{n-1} \cdot \ldots x_{2} \cdot x_{1}=G_{n}^{n},
\end{aligned}
$$

and thus $A_{n} \geq G_{n}$.

(AG) $\Longrightarrow(\mathrm{B})$. For $n=1$ we have even equality in (B). If $n \geq 2$ and $0<x \leq 1-\frac{1}{n}$, then $x^{n}>0 \geq 1+n(x-1)$, that is, (B) holds. Therefore, we can assume that $n \geq 2$ and $x>1-\frac{1}{n}$. Then $1+n(x-1)>0$, and now applying assumption (AG) to the $n$ positive numbers

$$
1+n(x-1), \overbrace{1,1, \ldots, 1}^{(n-1) \text { times }}
$$

we obtain

$$
\begin{aligned}
x^{n} & =\left\{\frac{[1+n(x-1)]+1+\ldots+1}{n}\right\}^{n} \\
& \geq[1+n(x-1)] \cdot 1 \cdot \ldots \cdot 1=1+n(x-1),
\end{aligned}
$$

and the inequality (B) is proved.

From the theorem, it follows that one way to get a simple proof of inequality (AG) is to give a simple proof of inequality (B). Here is one:

$$
\begin{aligned}
x^{n}-1-n(x-1) & =(x-1)\left(x^{n-1}+x^{n-2}+\ldots+1\right)-n(x-1) \\
& =(x-1)\left(x^{n-1}+x^{n-2}+\ldots+1-n\right) \geq 0 .
\end{aligned}
$$

The last inequality holds because the expression $x^{n-1}+$ $x^{n-2}+\ldots+1$ is bigger than $n$ for $x \geq 1$ and smaller than $n$ for $0<x \leq 1$. Thus (B) is proved, and consequently also $(A G)$. Other proofs of (AG) via inequality (B) were given in [6] and [3].

\section{REFERENCES}

[1] P. S. Bullen, D. S. Mitrinović, and P. M. Vasić, Means and their Inequalities, Reidel, Dordrecht, 1988.

[2] P. S. Bullen, Handbook of Means and their Inequalities, Kluwer, Dordrecht, 2003.

[3] M. D. Hirschhorn, The AM-GM Inequality, Math. Intelligencer 29, 7 (2007).

[4] L. Maligranda, Why Hölder's Inequality Should be Called Rogers' Inequality, Math. Inequal. Appl. 1, 69-83 (1998). 
[5] L. Maligranda, Bernoulli Inequality - Over 300 Years of History, in: Around Bernoullis, 19 $9^{\text {th }}$ All Polish Conference on History of Mathematics (June 6-10, 2005, Zamość), Lublin Technical University Publisher, Lublin, 2006, 31-62 (in Polish).

[6] D. Rüthing, Proofs of the Arithmetic Mean - Geometric Mean Inequality, Int. J. Math. Educ. Sci. Technology 13, 49-54 (1982).
Department of Engineering Sciences and Mathematics Luleå University of Technology

97187 Luleå

Sweden

e-mail: lech.maligranda@|tu.se 